\title{
ON TOPOLOGY INDUCED BY MEASURE
}

\section{P. A. FILLMORE}

Let $(X, \Re, \mu)$ be a complete $\sigma$-finite measure space with $\mu(X)>0$. The purpose of this note is to record the existence of a topology on $X$ with the property that each measurable (extended) real-valued function $f$ on $X$ is equal almost everywhere to a unique continuous (extended) real-valued function $f^{*}$ on $X$. The mapping $f \rightarrow f^{*}$ provides a "lifting," in the sense that it preserves the algebraic operations (where they are defined). The Stone-Čech compactification of this topology is also discussed.

If $\mathscr{K}$ denotes the $\sigma$-ideal in $\mathscr{N}$ of the sets of measure zero, then by a result of Maharam ([3]; see also [6]), there exists a mapping $\phi: \mathfrak{T} / \mathscr{N}$ $\rightarrow \mathfrak{N}$ such that

(1) $\phi(0)=\varnothing$ and $\phi(1)=X$,

(2) $\phi(p \cap q)=\phi(p) \cap \phi(q)$,

(3) $\phi(p \cup q)=\phi(p) \cup \phi(q)$,

(4) $\phi(p) \in p$,

for all $p, q \in \mathscr{M} / \mathscr{T}$. By (1) and (2) the sets $\phi(p)$ provide a basis for a topology on $X$, and this is the topology in question.

Theorem. Each continuous function on $X$ is measurable. For each measurable function $f$ on $X$, there exists a unique continuous function $f^{*}$ on $X$ which agrees almost everywhere with $f$. The mapping $f \rightarrow f^{*}$ preserves the algebraic operations (where they are defined).

Proof. For the first statement, it is sufficient to show that each open set is measurable. The following proof of this is taken from [3]. Any basic open set is measurable since the range of $\phi$ is in $\mathbb{T}$. Consider any family $\left\{\phi\left(p_{\alpha}\right)\right\}$ of basic open sets. Since the measure space is $\sigma$-finite, $\mathfrak{M} / \mathfrak{N}$ satisfies the countable chain condition and is a complete lattice. Hence $p=\bigcup_{p_{\alpha}}$ exists, and there is a sequence $\alpha_{1}, \alpha_{2}, \cdots$ of indices with $p=\bigcup_{p_{\alpha_{i}}}$. Since $\phi$ is monotone (by (2) or (3)),

$$
U \phi\left(p_{\alpha_{i}}\right) \subset U_{\phi\left(p_{\alpha}\right) \subset \phi(p) .}
$$

But $\phi\left(p_{\alpha_{i}}\right) \in p_{\alpha_{i}}$ by (4), so $U \phi\left(p_{\alpha_{i}}\right) \in U_{p_{\alpha_{i}}}=p$. Also $\phi(p) \in p$, hence $\phi(p)-U \phi\left(p_{\alpha_{i}}\right)$ is a null set, and therefore $\bigcup_{\phi}\left(p_{\alpha}\right)$ is measurable.

Now let $f$ be a measurable function on $X$, and define

$$
f^{*}(x)=\sup \left\{r \mid x \notin \phi\left[f^{-1}[-\infty, r)\right]\right\}
$$

Received by the editors December 20, 1965. 
for each $x \in X$. Here we are denoting by $[A]$ the element of $\mathscr{T} / \mathfrak{N}$ corresponding to $A \in \mathfrak{N}$. It is easy to verify that

$$
f^{*-1}[-\infty, r)=\bigcup_{s<r} \phi\left[f^{-1}[-\infty, s)\right]
$$

and

$$
f^{*-1}(r, \infty]=\underset{s<r}{\bigcup}\left(X-\phi\left[f^{-1}(-\infty, s]\right) .\right.
$$

Assumptions (1)-(3) imply that if $p^{\prime}$ is the complement of $p$, then $\phi\left(p^{\prime}\right)$ is the complement of $\phi(p)$, and therefore $f^{*}$ is continuous. Since the above unions need only be extended over rational $s$, the symmetric difference of $f^{*-1}[-\infty, r)$ and $f^{-1}[-\infty, r)$ is a null set for each real $r$. Consequently $f$ and $f^{*}$ differ only on a null set. For the uniqueness, it is sufficient to observe that each nonvoid open set contains a basic open set $\phi(p)$ with $p \neq 0$, and therefore has positive measure. That the algebraic operations are preserved is an immediate consequence of the uniqueness.

Our second observation concerns the Stone-Cech compactification of $X$. In order to discuss this, we introduce the Stone space $Y$ of the complete Boolean algebra $\mathscr{T} / \mathscr{T}$. The points of $Y$ are the ultrafilters (maximal dual ideals) $\mathcal{F}$ of $\mathfrak{T} / \mathfrak{T}$, and the basic open sets in $Y$ are the sets

$$
\gamma(p)=\{\mathfrak{F} \mid p \in \mathcal{F}\}
$$

as $p$ varies over $\mathfrak{T} / \mathscr{T}$. With this topology $Y$ is an extremally disconnected compact Hausdorff space, and the lattice $\mathfrak{T} / \mathscr{T}$ is isomorphic to the lattice of all open and closed subsets of $Y$. For each $x \in X$ define

$$
F(x)=\{p \mid p \in \mathscr{T} / \mathscr{N} \text { and } x \in \phi(p)\} .
$$

A similar construction is used by Donoghue in [1].

Lemma. $F(x)$ is an ultrafilter in $\mathfrak{T} / \mathfrak{T}$. The mapping $F: X \rightarrow Y$ satisfies $\phi=F^{-1} \circ \gamma$, and consequently the topology in $X$ is the weakest such that $F$ is continuous.

Proof. Since $\phi(0)=\varnothing$ by (1), we have $0 \notin F(x)$. On the other hand $\phi(1)=X$, so $1 \in F(x)$ for all $x$. If $p, q \in F(x)$, then $x \in \phi(p) \cap \phi(q)$ $=\phi(p \cap q)$ by (2), so $p \cap q \in F(x)$. Finally, if $p \in \mathfrak{N} / \mathscr{T}$ and $p^{\prime}$ is its complement, then

$$
\phi(p) \cup \phi\left(p^{\prime}\right)=\phi\left(p \cup p^{\prime}\right)=\phi(1)=X
$$

by (3) and (1). Thus either $x \in \phi(p)$ or $x \in \phi\left(p^{\prime}\right)$, so that either 
$p \in F(x)$ or $p^{\prime} \in F(x)$. Therefore $F(x)$ is an ultrafilter, and $F(x) \in Y$.

Concerning the second assertion of the lemma, for each $p \in \mathscr{T} / \mathfrak{N}$ and $x \in X$ the following statements are equivalent: $x \in\left(F^{-1} \circ \gamma\right)(p)$, $F(x) \in \gamma(p), p \in F(x)$, and $x \in \phi(p)$.

We remark that $X$ is a completely regular space, and that it is Hausdorff when $F$ is one-to-one.

TheOREM. Let $K$ be a compact Hausdorff space, and let $\alpha: X \rightarrow K$ be continuous. Then there exists a unique continuous $\bar{\alpha}: Y \rightarrow K$ such that $\alpha=\bar{\alpha} \circ F$.

Proof. We begin by observing that $F(X)$ is dense in $Y$. For if the basic open set $\gamma(p)$ is nonempty, then $p \neq 0, \phi(p)$ is nonempty, and $F(x) \in \gamma(p)$ for any $x \in \phi(p)$. Moreover, $Y$ is extremally disconnected, so that $F(X)$ is $C^{*}$-embedded [2, p. 96]. Therefore $Y$ is the StoneCech compactification of $F(X)$.

Next we note the existence of a continuous function $\beta: F(X) \rightarrow K$ such that $\alpha=\beta \circ F$. It follows easily from the lemma that $F\left(x_{1}\right)$ $=F\left(x_{2}\right)$ implies $\alpha\left(x_{1}\right)=\alpha\left(x_{2}\right)$, so that the function $\beta: F(x) \rightarrow \alpha(x)$ is well defined. The lemma also implies that $F$ is open in its range, so that if $U$ is an open subset of $K$, then $\beta^{-1}(U)=F\left(\alpha^{-1}(U)\right)$ is open. Thus $\beta$ is continuous.

Finally, by the first paragraph of the proof, $\beta$ has a continuous extension $\bar{\alpha}$ to all of $Y$, and clearly $\alpha=\bar{\alpha} \circ F$. The density of $F(X)$ implies the uniqueness assertion, and completes the proof.

REMARKS. 1. A proof of the above theorem may be based on the following construction. If $g$ is a continuous (extended) real-valued function on $X$, let

$$
\bar{g}(\mathcal{F})=\sup \left\{r \mid \mathcal{F} \notin \gamma\left[g^{-1}[-\infty, r)\right]\right\}
$$

for each $F \in Y$. Then $\bar{g}$ is a continuous function on $Y$ satisfying $g=\bar{g} \circ F$. If $g$ is merely measurable, then $g^{*}=\bar{g} \circ F$ is the continuous function constructed in the first theorem.

2. The mapping $f \rightarrow\left(f^{*}\right)^{-}$from $L^{\infty}(X, \mathfrak{T}, \mu)$ into $C(Y)$ is easily seen to be an isometric isomorphism, and is in fact the Gelfand transform.

3. In [7] the topology with basis consisting of the sets $\phi(p)-N$, where $N \in \eta$, is considered. This is stronger than the above topology, but admits no more continuous functions.

4. Those measure spaces for which a mapping $\phi$ satisfying (1)-(4) exists are characterized in [4]. If the space is not $\sigma$-finite, then a continuous function may fail to be measurable, but the remainder of the 
first theorem is valid in this context. For the second theorem the completeness of $\mathfrak{T} / \mathfrak{N}$ appears essential.

\section{REFERENCES}

1. W. F. Donoghue, Jr., On the lifting property, Proc. Amer. Math. Soc. 16(1965), 913-914.

2. L. Gillman and M. Jerson, Rings of continuous functions, Van Nostrand, New York, 1960.

3. D. Maharam, On a theorem of von Neumann, Proc. Amer. Math. Soc. 9 (1958), 987-994.

4. R. Ryan, Representative sets and direct sums, Proc. Amer. Math. Soc. 15 (1964), 387-390.

5. R. Sikorski, Boolean algebras, 2nd ed., Academic Press, New York, 1964.

6. A. Ionescu Tulcea and C. Ionescu Tulcea, On the lifting property (I), J. Math. Anal. Appl. 3 (1961), 537-546.

7. - On the lifting property (IV), Ann. Inst. Fourier (Grenoble) 14 (1964), $445-472$.

INDIANA UNIVERSITY 